\title{
Cusp height in aortic valves
}

\author{
Hans-Joachim Schäfers, MD, ${ }^{\mathrm{a}}$ Wolfram Schmied, Dipl Psych, ${ }^{\mathrm{a}}$ Gil Marom, MSc, ${ }^{\mathrm{b}}$ and Diana Aicher, $\mathrm{MD}^{\mathrm{a}}$
}

Objectives: Successful aortic valve repair must normalize cusp and root dimensions. Limited information is available on the normal dimensions of human cusps, in particular the cusp height.

\begin{abstract}
Methods: The cusp height was measured intraoperatively in 621 patients during aortic valve repair procedures. A tricuspid anatomy was present in 329 patients and bicuspid in 286 patients. In addition, patient age, gender, height, weight, preoperative degree of aortic regurgitation, and aortic dimensions were recorded. The data were analyzed for possible interrelation between the cusp height and clinical variables.

Results: In the bicuspid valves, the geometric height of the nonfused cusp ranged from 15 to $30 \mathrm{~mm}$ (mean, 23.8 \pm 2.0 ). Significant correlations were found between the cusp height and all clinical variables. In the tricuspid valves, the height of the noncoronary cusp ranged from 14 to $28 \mathrm{~mm}$ (mean, $20.7 \pm 2.2$ ). The height of the left coronary cusp varied from 12 to $25 \mathrm{~mm}$ (mean, $20.0 \pm 2.1$ ) and that of the right coronary cusp from 12 to $25 \mathrm{~mm}$ (mean, $20.0 \pm 2.1$ ). The noncoronary cusp was significantly greater than the left and the right coronary cusp $(P=.000)$. No difference was found between the left and right cusps $(P=.513)$. Significant correlations between the geometric height and clinical parameters were found for most clinical variables, excluding the degree of aortic regurgitation.
\end{abstract}

Conclusions: We found the cusp height was larger than previously published. It shows marked variability and correlates with the clinical variables. These data might serve as the basis for decision making in aortic valve repair. (J Thorac Cardiovasc Surg 2013;146:269-74)

In the past 2 decades, valve-preserving aortic replacement and valve repair have become increasingly popular alternatives to aortic valve replacement for tricuspid and bicuspid valve anatomy. The geometric interrelation between the cusps and root as a functional unit ${ }^{1,2}$ has led to the realization that near-normal cusp and root geometry is essential for a good functional result of repair.

Similar to Swanson and Clark, ${ }^{3}$ we found that the configuration of the human aortic valve follows a constant pattern. ${ }^{4,5}$ The initial assumption was that the height difference between the aortoventricular plane and the free margins of the cusps in diastole (effective height) could be used as indicator of valve configuration. ${ }^{4}$ In an echocardiographic study and a numeric model, we were able to confirm the hypothesis and its importance in aortic valve repair. ${ }^{5,6}$ At that time, we noted that the effective height could only be used as an indicator of cusp prolapse if cusp retraction was absent (ie, tissue height or geometric height) were normal. ${ }^{5}$ The data indicate that in

\footnotetext{
From the Department of Thoracic and Cardiovascular Surgery, ${ }^{a}$ University Hospitals of Saarland, Homburg/Saar, Germany; and School of Mechanical Engineering, ${ }^{\text {b }}$ Tel Aviv University Faculty of Engineering, Tel Aviv, Israel.

Disclosures: Authors have nothing to disclose with regard to commercial support.

Received for publication Feb 13, 2012; revisions received May 27, 2012; accepted for publication June 18, 2012; available ahead of print Aug 2, 2012.

Address for reprints: Hans-Joachim Schäfers, MD, Department of Thoracic and Cardiovascular Surgery, University Hospitals of Saarland, Homburg/Saar, Germany (E-mail: h-j.schaefers@uks.eu).

$0022-5223 / \$ 36.00$

Copyright (c) 2013 by The American Association for Thoracic Surgery http://dx.doi.org/10.1016/j.jtcvs.2012.06.053
}

a normally functioning aortic valve, the relationship should be fixed among the cusp height, effective height, and root dimensions. It appears as self-explanatory that the applicability of the concept relies on a normal amount of cusp tissue.

The amount of cusp tissue is by definition decreased in diseases that lead to cusp retraction. This can occur as a consequence of aging and inflammatory valve disease and seems to predominantly involve cusp tissue height. To date, repair procedures for retracted cusps have not resulted in good valve stability, ${ }^{7}$ indicating this pathologic entity requires a specific approach. Thus, the determination of cusp height should be of clinical relevance for aortic valve reconstruction, not only in judging the possibility of repair, but also in defining the surgical strategy to achieve a normal aortic valve configuration.

Limited information is available regarding the normal human cusp dimensions. ${ }^{3,8-11}$ Three studies were performed of a very limited number of aortic valves, ${ }^{3,8,9}$ another two were performed on formalin-preserved hearts obtained at autopsy. ${ }^{10,11}$ The exact determination of the cusp height was not defined in those reports, and no attempt was made to place the cusp height in the context of possible interindividual differences, such as valve anatomy, gender, or body size. Our own clinical observations indicated that the geometric height of aortic cusps can vary and, in general, could be higher than published previously. ${ }^{3,8,10,11}$ It appeared reasonable to assume that the cusp size could possibly relate to the body size, such as has been found for the aortic and pulmonary valve diameters. ${ }^{12}$ 


\section{Abbreviations and Acronyms \\ $\mathrm{BAV}=$ bicuspid aortic valve \\ $\mathrm{TAV}=$ tricuspid aortic valve \\ TEE $=$ transesophageal echocardiography}

In a prospective study, we measured the geometric height of the aortic cusps during aortic repair procedures to determine the normal values for tricuspid aortic valves (TAVs) and bicuspid aortic valves (BAVs). In addition, we analyzed the data for possible correlations with clinical information relating to the patient characteristics.

\section{METHODS}

From April 2003 to April 2011, the cusp geometric height (Figure 1) was measured and documented prospectively in every aortic repair procedure, involving bicuspid or tricuspid morphology $(n=615)$. The preoperative dysfunction of the aortic valve ranged from trivial to severe, depending on the indication for surgery (aortic root aneurysm or severity of aortic regurgitation with clinical symptoms).

The conditions were standardized in all patients. The maximum sinus dimensions and sinotubular and aortoventricular dimensions were determined by transesophageal echocardiography (TEE) before cardiopulmonary bypass. The clinical data were collected. The data were analyzed retrospectively. For the purposes of the present study, only patients were included who had bicuspid or tricuspid morphology of their aortic valves. Thus, patients with unicuspid or quadricuspid aortic valve anatomy were excluded. All patients gave consent to collection and analysis of the data in anonymized fashion. The local ethics committee approved the study.

After crossclamping, the aorta was opened by a transverse incision approximately $1 \mathrm{~cm}$ above the sinotubular junction and cardioplegia given. The aortoventricular diameter was measured by intubation with graded Hegar dilators. The commissures were placed under radial tension using stay sutures. The free margin of the cusp to be measured was held with a forceps and placed under tension. The geometric height was measured with a ruler from the aortic insertion in the nadir of the sinus to the nodulus of Arantius of the respective cusp (Figure 2). This measurement was repeated, and the data were only used if the 2 dimensions were identical. This was the case in all instances. In the TAVs, all 3 cusps were measured. In the BAVs, only the geometric height of the nonfused cusp was determined. Because of the variable pattern of fusion in the bicuspid anatomy, we believed measurements on the fused cusps would not generate relevant information. From the initial observations, the repair procedure was pursued in most patients if a geometric height of $17 \mathrm{~mm}$ or more in TAVs and $20 \mathrm{~mm}$ or more in BAVs was found. If the valve was replaced, the individual data were not recorded. Thus, by this definition, 12 patients with TAVs and 3 patients with BAVs underwent replacement for apparent cusp retraction and they were not included in the present study. In 13 patients with tricuspid and 3 patients with bicuspid morphology, the repair was pursued because of their young age and strong wish to avoid anticoagulation.

The data were analyzed retrospectively to define the normal values for BAVs and TAVs. In addition, we attempted to determine whether correlations could be found between the geometric height and other clinical parameters.

The parameters were gender, age, height, weight, body surface area, preoperative degree of aortic regurgitation, aortoventricular and sinus diameters determined by echocardiography in diastole, and the aortoventricular diameter measured by direct intubation. In the BAVs, the type and degree of fusion (partial, $\mathrm{n}=111$ vs complete, $\mathrm{n}=175$ ) were documented and analyzed.
Measurements were made in 615 patients, 329 with TAVs and 286 with BAVs. Because in those with TAVs, all 3 cusps, and in those with BAVs, only the nonfused cusps were measured, a total of 1273 measurements was obtained.

Cusp repair procedures for prolapse were performed in 614 patients of this cohort ( 328 with TAVs and 286 with BAVs). In the 328 patients with TAVs, the procedures included plication of the free cusp margin $(n=461)$, triangular resection $(n=18)$, and insertion of a pericardial patch $(\mathrm{n}=107)$. The $286 \mathrm{BAV}$ cusp procedures included plication of the free cusp margin $(n=257)$, triangular resection $(n=92)$, and pericardial patch insertion $(n=82)$. These data were not analyzed further, because they were beyond the scope of the present study.

\section{Biscuspid Aortic Valves}

Of the 286 patients with BAVs, $254(88.8 \%)$ were men. Their age ranged from 18 to 86 years (mean, $46.6 \pm 13.4$; median, 45.0 ). The body height varied from 149 to $198 \mathrm{~cm}$ (mean, $178.5 \pm 8.5$; median, 178.0). The body weight was 46 to $132 \mathrm{~kg}$ (mean, $86.7 \pm 14.7$; median, 85.0), the body surface area ranged from 1.41 to $2.66 \mathrm{~m}^{2}$ (mean, $2.07 \pm 0.21$; median, 2.05). Using TEE, the aortoventricular diameter varied from 16 to 40 $\mathrm{mm}$ (mean, $29.7 \pm 3.2$; median, 29.0), the sinus diameter varied from 19 to $74 \mathrm{~mm}$ (mean, $40.8 \pm 7.4$; median, 39.5), and the sinotubular diameter varied from 12 to $50 \mathrm{~mm}$ (mean, $31.0 \pm 5.8$; median, 29.5). The aortoventricular diameter, as determined by intubation with a Hegar, ranged from 21 to $40 \mathrm{~mm}$ (mean, $31.8 \pm 2.9$; median, 32.0). Complete fusion was present in 175 cases. The nonfused cusp was noncoronary in 248 , the right coronary in 36 , and the left coronary in 2 cases.

Minimal or mild regurgitation was present in 51 and moderate or severe regurgitation in 235 cases. An initial analysis compared the geometric height of the 3 nonfused cusps in the 3 different types of fusion. Additional calculations regarding the correlations between geometric height and other parameters were performed, summarizing the fusion types and using the respective nonfused cusp.

\section{Tricuspid Aortic Valves}

Of the 329 patients with TAVs, 237 (72.0\%) were men. Their age ranged from 18 to 84 years (mean, $60.4 \pm 13.2$; median, 63.0). The body height varied from 147 to $207 \mathrm{~cm}$ (mean, $174.8 \pm 10.0$; median, 175.0), the body weight from 40 to $140 \mathrm{~kg}$ (mean, $83.0 \pm 16.1$; median, 82.0). The body surface area ranged from 1.28 to $2.81 \mathrm{~m}^{2}$ (mean, $2.0 \pm 0.23$; median, 2.0). Using TEE, the aortoventricular diameter ranged from 20 to 35 $\mathrm{mm}$ (mean, 27.6 \pm 2.8 ; median, 27.0), the sinus diameter varied from 25 to $81 \mathrm{~mm}$ (mean, $44.2 \pm 9.8$; median, 41.0), and the sinotubular diameter from 22 to $51 \mathrm{~mm}$ (mean, $34.5 \pm 7.1$; median, 33.0). The aortoventricular, as diameter determined by intubation with a Hegar, ranged from 24 to 35 $\mathrm{mm}$ (mean, $30.2 \pm 2.4$; median, 30.0). An initial analysis compared the geometric height of the 3 cusps. Additional calculations regarding the correlations between the geometric height and other parameters were performed with the mean of the 3 cusps. Minimal or mild regurgitation was present in 55 and moderate or severe regurgitation in 274 cases.

\section{Statistical Analysis}

Data analysis was performed using IBM SPSS Statistics, version 19 (SPSS, IBM, Somers, NY). Descriptive statistics are expressed as the mean \pm standard deviation. The normal distribution of cusp height in the BAVs and TAVs was analyzed using the Shapiro-Wilk test. Although the data were not normally distributed, parametric tests were used for additional analysis because of the large sample sizes. Correlations were assessed using Pearson's correlation coefficient. In the BAVs, the differences depending on the degree of fusion (partial vs complete) were analyzed using $t$-tests. Differences regarding the type of fusion were tested with analysis of variance, followed by the Scheffé test as post hoc testing. $P$ values $<.05$ were considered statistically significant. 


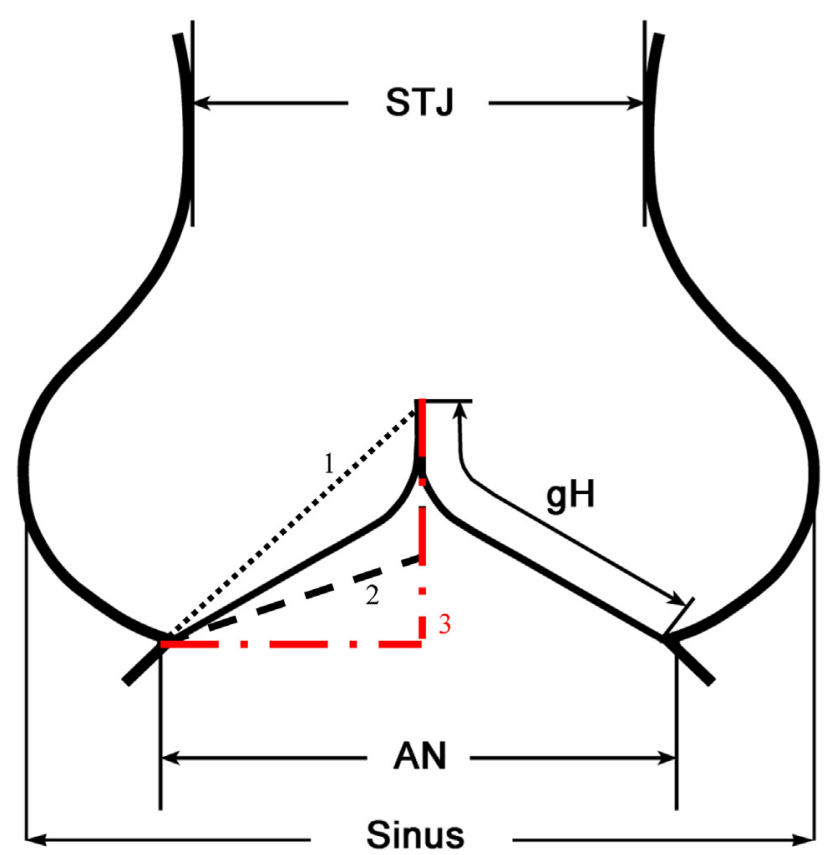

FIGURE 1. Schematic drawing of the aortic root with graphic description of geometric height. $A N$, Aortoventricular junction; $g H$, geometric height; STJ, sinotubular junction; sinus, maximal sinus diameter; 1 , shortest distance from aortic insertion to coaptation line; 2, distance assuming a straight course of the cusp and a coaptation height of $4 \mathrm{~mm} ; 3$, maximum geometric height assuming the effective height is equal to the coaptation height.

\section{RESULTS}

\section{Bicuspid Aortic Valves}

The geometric height of the nonfused cusp ranged from 15 to $30 \mathrm{~mm}$ (mean, $23.8 \pm 2.0$; median, 24.0; Figure 3). In 26 patients with no more than mild regurgitation and preserved sinus dimensions $(<40 \mathrm{~mm})$, a height of $22.7 \pm 1.9$ $\mathrm{mm}$ was obtained. Significant differences were found between men and women $(24.1 \pm 1.9$ vs $22.2 \pm 2.2 \mathrm{~mm}$; $P<.001)$. For the different types of fusion, no significant difference $(P=.081)$ was found between the heights of

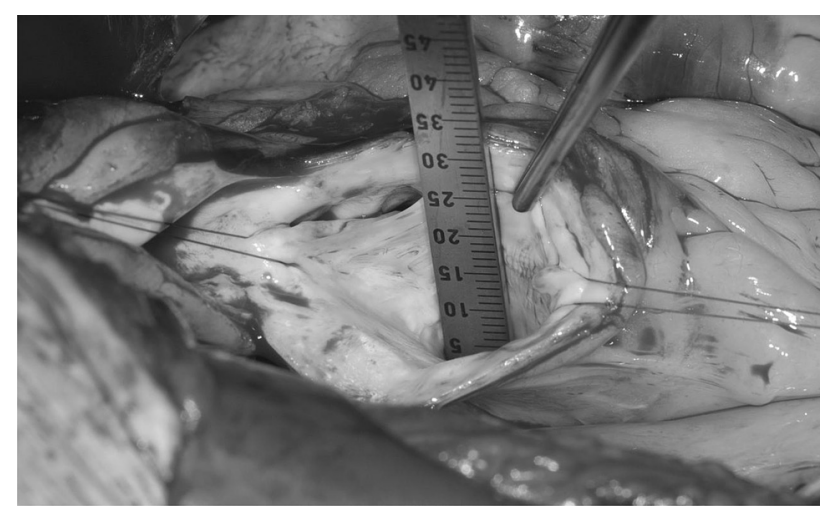

FIGURE 2. Intraoperative photograph showing measurement of geometric height.

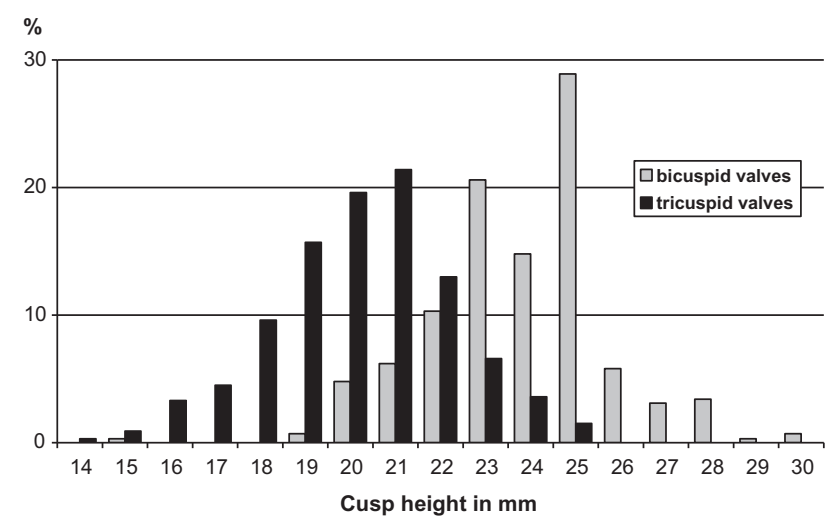

FIGURE 3. Distribution of geometric height in bicuspid $(\mathrm{n}=289$; nonfused cusps) and tricuspid ( $\mathrm{n}=332$; mean of all 3 cusps) aortic valves.

the noncoronary (mean, $23.9 \pm 2.0 \mathrm{~mm}$; range, 15-30; $\mathrm{n}=248$ ), left coronary (mean, $23.3 \pm 2.0 \mathrm{~mm}$; range, $19-27 ; \mathrm{n}=36)$, or right coronary $(26.0 \pm 5.7 \mathrm{~mm}$; range, 22-30; $\mathrm{n}=2$ ) cusps.

The difference in the geometric height of the nonfused cusp between those with complete fusion $(24.1 \pm 1.8$ $\mathrm{mm})$ and those with partial fusion $(23.4 \pm 2.3 \mathrm{~mm})$ was small, but significant $(P=.013)$. In patients with minimal or mild aortic regurgitation, the geometric height was $23.0 \pm 1.8 \mathrm{~mm}$, and in those with moderate or severe regurgitation, it was $24.0 \pm 2.0 \mathrm{~mm}(P=.001)$.

Significant-although weak-correlations between the geometric height and clinical parameters were found for most variables (Table 1). Relevant correlations were found between the geometric height and the aortoventricular junction $(r=0.36 ; P<.001)$ and body height $(r=0.31$; $P<.001)$.

\section{Tricuspid Aortic Valves}

The geometric height of the noncoronary cusp ranged from 14 to $28 \mathrm{~mm}$ (mean, $20.7 \pm 2.2$; median, 20.0). In 28 patients

TABLE 1. Correlation coefficients between geometric height and different parameters

\begin{tabular}{|c|c|c|c|c|}
\hline \multirow[b]{2}{*}{ Variable } & \multicolumn{2}{|c|}{ BAVs } & \multicolumn{2}{|c|}{ TAVs } \\
\hline & $\boldsymbol{r}$ & $P$ Value & $\boldsymbol{r}$ & $P$ value \\
\hline Age & -0.263 & .000 & -0.155 & .005 \\
\hline Female gender & -0.290 & .000 & -0.520 & .000 \\
\hline Body height & 0.305 & .000 & 0.472 & .000 \\
\hline Body weight & 0.150 & .012 & 0.391 & .000 \\
\hline BSA & 0.202 & .001 & 0.444 & .000 \\
\hline BMI & -0.023 & .703 & 0.151 & .007 \\
\hline Preoperative AR & 0.248 & .000 & -0.075 & .172 \\
\hline AV junction (TEE) & 0.363 & .000 & 0.584 & .000 \\
\hline AV junction (intubation) & 0.360 & .000 & 0.530 & .000 \\
\hline Sinus diameter & 0.201 & .002 & 0.492 & .000 \\
\hline STJ diameter & 0.196 & .002 & 0.343 & .000 \\
\hline
\end{tabular}

$A R$, Aortic regurgitation; $B A V$, bicuspid aortic valves; $B M I$, body mass index; $B S A$ body surface area; $S T J$, sinotubular; $T A V$, tricuspid aortic valves; $T E E$, transesophageal echocardiography; $A V$, atrioventricular. 


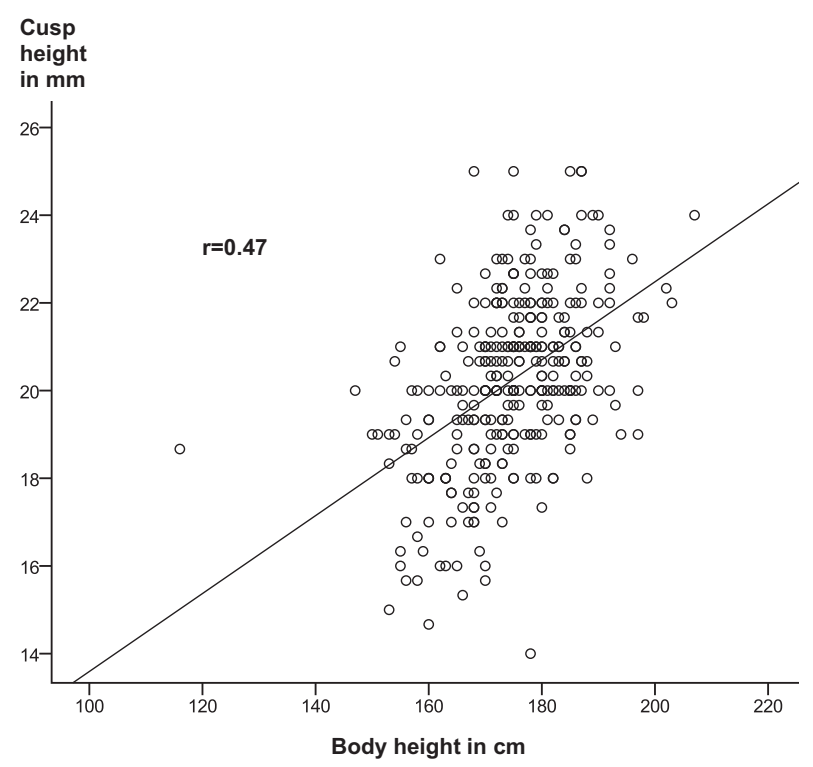

FIGURE 4. Correlation between body height and geometric height in tricuspid aortic valves ( $\mathrm{n}=332$; mean of all 3 cusps).

with no more than mild regurgitation and preserved sinus dimensions ( $<40 \mathrm{~mm}$ ), a geometric height of $20.0 \pm 2.4 \mathrm{~mm}$ was obtained. The geometric height of the left coronary cusp varied from 12 to $25 \mathrm{~mm}$ (mean, $20.0 \pm 2.1$; median, 20.0) and that of the right coronary cusp from 12 to $25 \mathrm{~mm}$ (mean, $20.0 \pm 2.1$; median, 20.0). The noncoronary cusp height was significantly greater than that of the left and the right coronary cusps $(P<.001)$; no difference was found between the left and right cusps $(P=.513)$.

Similar to the BAVs, the cusps were larger in the men than in the women for all 3 cusps (noncoronary, $21.3 \pm$ $1.9 \mathrm{~mm}$ vs $19.1 \pm 2.3 \mathrm{~mm}, P<.001$; right coronary, 20.6 $\pm 1.8 \mathrm{~mm}$ vs $18.2 \pm 2.0 \mathrm{~mm}, P<.001$; left coronary, $20.7 \pm 1.8 \mathrm{~mm}$ vs $18.3 \pm 2.0 \mathrm{~mm}, P<.001)$. In patients with minimal or mild aortic regurgitation, the geometric height was $20.5 \pm 2.0 \mathrm{~mm}(\mathrm{n}=55)$, and in those with moderate or severe regurgitation, it was $20.1 \pm 2.0 \mathrm{~mm}$ (n $=274 ; P=.217$ ).

Significant correlations between the geometric height and clinical parameters were found for body height (Figure 4; $r=0.47)$, weight $(r=0.39)$, body surface area $(r=0.44)$, sinus diameter $(r=0.49)$, and aortoventricular diameter ( $r=0.58$ and $r=0.53$; each $P<.001$; Table 1 ). A significant, although weak, negative correlation was found between the geometric height and age $(r=-0.16 ; P=.005)$.

\section{DISCUSSION}

Quite obviously, the aortic cusp configuration depends on the root dimensions and cusp size. Normal values of the root dimensions have been obtained by $\operatorname{direct}^{3,5,8,11}$ and echocardiographic $^{12-14}$ examinations. Less is known about the normal data for cusp dimensions (ie, the cusp width and height). Measurements have been performed on TAVs, but not BAVs. Because the aortoventricular and sinotubular dimensions vary with clinical parameters such as age and body height, one would expect a similar variation in the geometric cusp size. The cusp size is a function of the width and height. The width is usually determined by direct or echocardiographic measurement of the length of the free margin, which has been done in a few ex vivo studies. $^{3,8-11}$ The length of the free margin is certainly important information; however, we have found it very difficult to measure it exactly in vivo (ie, intraoperatively).

The cusp height is the other parameter that determines the cusp size. This height can be calculated as an integral of the distances between the aortic insertion and free margin if a full geometric representation of the cusp is available. The maximum cusp height (ie, the largest distance between the aortic insertion and free margin in the center of the cusp), can be assumed as an indicator of the cumulative cusp height. At this point, there is limited and somewhat confusing information on the normal height of a human cusp, and different terms have been used for this dimension. To avoid confusion in terminology and to differentiate it from effective height (ie, from the basal plane to the central coaptation level), we have proposed to call the maximum tissue height the "geometric height" of the cusp. It can be measured easily in the operating room, and the data we have collected have indicated a relatively low variability, indicating a high reproducibility. Because the aortoventricular and sinotubular dimensions vary with clinical parameters such as age and body height, one would expect a similar variation in the geometric cusp height. This variability was confirmed by our present results.

Swanson and Clark (3) analyzed 8 hearts obtained at autopsy within 3 days of death. The hearts were cooled until the investigation and then studied under different pressures. They expressed the cusp height as a percentage of the aortoventricular junction and reported a height of $70 \%$ of the annular size, which would translate to $17 \mathrm{~mm}$. The variability of the different measurements was not specified. Kunzelman and colleagues ${ }^{8}$ studied 10 cryopreserved homografts with TAVs obtained from a tissue bank. Nine valves were from male donors, with an age range of 18 to 54 years. They measured the geometric height of the cusps, although they did not specify whether the cusps were stretched to mimic physiologic conditions. They found an average cusp height of the 3 cusps of approximately $14 \mathrm{~mm}$, with limited variability. Thubrikar and colleagues ${ }^{9}$ investigated 14 aortic valves in patients undergoing surgery for aortic regurgitation ( 9 men) and determined the cusp dimensions from TEE. They found a wide variation in the cusp height (range, 13 to $25 \mathrm{~mm}$; mean, 19.6).

Vollebergh and Becker ${ }^{10}$ analyzed 200 hearts from routine autopsies with an age range of 7 to 93 years. They did not specify how the hearts were preserved or under 
what conditions the measurements were made. The primary goal was the analysis of the cusp width; however, it is unclear whether they meant the length of the free margin or the intercommissural distance. They reported a mean cusp height of $14 \mathrm{~mm}$ in all 3 cusps. Silver and Roberts ${ }^{11}$ studied the cusp dimensions in 100 hearts that had been fixed in formalin after autopsy. By selecting only noncardiac or coronary causes of death, they concluded that all aortic valves had functioned normally. They analyzed the relationship between the heart weight and cusp height (mean, $15 \mathrm{~mm}$ ) but did not study the relationship between the cusp size and clinical parameters such as gender, subject height, or weight.

Our current data revealed values that were consistently greater than most data reported previously., ${ }^{3,10,11}$ Although these 4 reports analyzed the cusp height, the conditions of the measurement were not defined in the publications and were probably not uniform. Cusps will crimp when not placed under pressure, and the conditions of measurement could have led to an underestimation of the true tissue height. In addition, fixation in formalin could lead to tissue retraction, which might explain why Swanson and Clark $^{3}$ found a greater cusp height than did the 2 autopsy series. ${ }^{9,10}$

We consistently extended the cusp by traction with a forceps, minimizing or eliminating the aspect of crimping. Finally, some of the values published for geometric cusp height do not appear geometrically plausible. If one assumes an aortoventricular diameter (a) of $22 \mathrm{~mm}$ and an effective height of $9 \mathrm{~mm}$ (b) and applies Pythagoras' theorem $\left(a^{2}+b^{2}=c^{2}\right)$, the cusp geometric height (c) would have to be at least $14.2 \mathrm{~mm}(\mathrm{c}=\sqrt{ } 202)$. This, however, would imply that the cusps are not curved and simply touch at the free margins without coaptation overlap. ${ }^{3}$ For an effective height of $10 \mathrm{~mm}$, which is not unusual, ${ }^{5}$ the geometric height would be calculated to be at least $14.8 \mathrm{~mm}$ (Figure 1, dashed line 1). If one assumes, more realistically, an initial straight line to the center with a coaptation height of $3 \mathrm{~mm}$ (3), the minimum geometric height would be at least $16 \mathrm{~mm}(13+3 \mathrm{~mm}$; Figure 1, dashed line 2). These assumptions would only apply for the size of the aortoventricular junction that is on the lower end of the measured sizes. ${ }^{11,13-15}$ Thus, of the published data, only those from Swanson and Clark ${ }^{3}$ and Thubrikar and colleagues $^{9}$ appear geometrically plausible. Our data on TAVs are comparable with the data from the latter 2 studies. Furthermore, the maximum geometric height of nonprolapsing cusp would be $21 \mathrm{~mm}(10+11 \mathrm{~mm})$, assuming the effective height is equal to the coaptation height (Figure 1, dashed line 3). Obviously, the height would be larger if the cusp were enlarged and prolapsing. Although this is a rough approximation of the natural cusp configuration, this height is closer to the current results than the previous estimates.
Our key finding was the wide distribution of cusp height, with correlations found with gender and body height and less with other parameters. This observation is in line with the variability found for root dimensions described in other studies. ${ }^{12-16}$ These clinical parameters have not been previously analyzed and, thus, a direct comparison of our results with those from the other reports ${ }^{3,8-11}$ was not possible.

As could be expected, a positive correlation was found between the geometric cusp height and body height (which is also connected to body size or male gender). We also found a weak correlation between the maximum root diameter and geometric cusp height, which corresponds to the findings of Thubrikar and colleagues. ${ }^{9}$ The reason for this relationship is still unclear. It might be due to congenital factors (ie, predetermination of larger cusps in patients with a tendency to have a larger root) or it might be due to a remodeling effect of the cusp size with increasing root dimensions. ${ }^{9}$ Because our study had a cross-sectional character, it was impossible to differentiate between these 2 possible mechanisms.

From our clinical observations, it was not surprising that the size of the nonfused cusps in BAVs larger than the cusps of TAVs. We found a similar distribution with correlations with patient characteristics. These, however, tended to be lower than in those in the TAV anatomy. The type of fusion (partial vs complete) did not seem to have an effect on the cusp size. The lower correlations with other patient data indicate the possibility of an influence of other factors that were not assessed in the present investigation, including the anatomic variability found in bicuspid anatomy. ${ }^{17}$

The current data might have immediate implications on the clinical application of aortic valve reconstruction. Repair procedures for retracted cusps have not resulted in good valve stability, ${ }^{7}$ and we have made similar observations. To date, a clinical definition of retracted cusps has been lacking. From the results of our measurements, we have arbitrarily defined retraction in the adult as a cusp height of $16 \mathrm{~mm}$ or less in TAVs and $19 \mathrm{~mm}$ or less in the BAVs. These arbitrary cutoff values led to defining $1 \%$ of the bicuspid and $4 \%$ of the tricuspid cusps as retracted. In these cases, we mostly aborted the repair and chose valve replacement. Of the 15 patients in the present cohort who underwent repair with a geometric height less than the proposed cutoff values, 2 required reoperation within the first 2 postoperative years.

The limitations of the present study included that the patients by definition had either an abnormally large aorta or relevant aortic regurgitation. We did not study normal aortic valves in the presence of normal aortic dimensions. However, such as study would be difficult to justify. One can assume that the effect of these 2 aspects would be relatively small, because the degree of preoperative aortic regurgitation did not have a relevant relation to geometric cusp height. 


\section{CONCLUSIONS}

The cusp geometric height that we found was larger than that previously published. It showed some variability and correlated with the clinical variables. The nonfused cusps of the BAVs were $3 \mathrm{~mm}$ larger than those of the TAVs. These data could serve as a basis for decision making in aortic valve repair and serve as a reference for computer simulation studies of the aortic valve.

\section{References}

1. Langer F, Aicher D, Kissinger A, Wendler O, Lausberg H, Fries R, et al. Aortic valve repair using a differentiated surgical strategy. Circulation. 2004;110(suppl 1):II67-73.

2. Boodhwani M, de Kerchove L, Glineur D, Poncelet A, Rubay J, Astarci P, et al. Repair-oriented classification of aortic insufficiency: impact on surgical techniques and clinical outcomes. J Thorac Cardiovasc Surg. 2009;137:286-94

3. Swanson WM, Clark RE. Aortic valve leaflet motion during systole: numericalgraphical determination. Circ Res. 1973;32:42-8.

4. Schäfers HJ, Bierbach B, Aicher D. A new approach to the assessment of aortic cusp geometry. J Thorac Cardiovasc Surg. 2006;132:436-8.

5. Bierbach B, Aicher D, Issa OA, Bomberg H, Gräber S, Glombitza P, et al. Aortic root and cusp configuration determine aortic valve function. Eur J Cardiothorac Surg. 2010;38:400-6.

6. Marom G, Haj-Ali R, Rosenfeld M, Schäfers HJ, Raanani E. Aortic root numerical model: annulus diameter prediction of effective height and coaptation in post aortic valve repair. J Thorac Cardiovasc Surg Epub 2012 Feb 23.

7. Le Polain de Waroux JB, Pouleur AC, Robert A, Pasquet A, Gerber BL, Noirhomme $\mathrm{P}$, et al. Mechanisms of recurrent aortic regurgitation after aortic valve repair: predictive value of intraoperative transesophageal echocardiography. JACC Cardiovasc Imaging. 2009;931-9.

8. Kunzelman KS, Grande KJ, David TE, Cochran RP, Verrier ED. Aortic root and valve relationships: impact on surgical repair. J Thorac Cardiovasc Surg. 1994; 107:162-70.

9. Thubrikar MJ, Labrosse MR, Zehr KJ, Robicsek F, Gong GG, Fowler BL. Aortic root dilatation may alter the dimensions of the valve leaflets. Eur J Cardiothorac Surg. 2005;28:850-5.

10. Vollebergh FE, Becker AE. Minor congenital variations of cusp size in tricuspid aortic valves: possible link with isolated aortic stenosis. Br Heart J. 1977;39: 1006-11.

11. Silver MA, Roberts WC. Detailed anatomy of the normally functioning aortic valve in hearts of normal and increased weight. Am J Cardiol. 1985;55: 454-61.

12. Capps SB, Elkins RC, Fronk DM. Body surface area as a predictor of aortic and pulmonary valve diameter. J Thorac Cardiovasc Surg. 2000;119:975-82.

13. Sheil ML, Jenkins O, Sholler GF. Echocardiographic assessment of aortic root dimensions in normal children based on measurement of a new ratio of aortic size independent of growth. Am J Cardiol. 1995;75:711-5.

14. Babaee Bigi MA, Aslani A. Aortic root size and prevalence of aortic regurgitation in elite strength trained athletes. Am J Cardiol. 2007;100: 528-30.

15. La Canna G, Maisano F, De Michele L, Grimaldi A, Grassi F, Capritti E, et al. Determinants of the degree of functional aortic regurgitation in patients with anatomically normal aortic valve and ascending thoracic aorta aneurysm. Transoesophageal Doppler echocardiography study. Heart. 2009;95:130-6.

16. Zhu D, Zhao Q. Dynamic normal aortic root diameters: implications for aortic root reconstruction. Ann Thorac Surg. 2011;91:485-90.

17. Sabet HY, Edwards WD, Tazelaar HD, Daly RC. Congenitally bicuspid aortic valves: a surgical pathology study of 542 cases (1991 through 1996) and a literature review of 2,715 additional cases. Mayo Clin Proc. $1999 ; 74: 14-26$ 